\title{
Mycotic Aneurysm of the Aortic Arch
}

Ji Hye Seo, M.D., Dae Won Park, M.D., and Won Suk Choi, M.D., Ph.D.

Division of Infectious Diseases, Department of Internal Medicine, Korea University College of Medicine, Seoul, Korea

A mycotic aneurysm of the thoracic aorta is rare. We report a case of mycotic aneurysm that developed in the aortic arch. An 86-year-old man was admitted with fever and general weakness. Blood culture yielded methicillin-resistant Staphylococcus aureus. Chest X-ray showed an enlarged aortic arch, and computed tomography scan revealed an aneurysm in the aortic arch. The patient was treated only with antibiotics and not surgically. The size of the aneurysm increased rapidly, resulting in bronchial obstruction and superimposed pneumonia. The patient died of respiratory failure.

Key Words: aortic arch; infected aneurysm; Staphylococcus aureus.

Mycotic aneurysm is arterial aneurysm of infectious etiology independent of the causative microorganism, with the exception of syphilitic aortitis. The most common infection sites are the femoral artery and the abdominal aorta.[1,2] Mycotic aneurysm of the thoracic aorta is extremely rare. We report a case of mycotic aneurysm in the aortic arch, which had a fatal clinical course.

\section{Case Report}

An 86-year-old man was admitted to our hospital with fever and general weakness in August 2012. He had hypertension and chronic kidney disease. Hemodialysis had been initiated in May 2013 through a permanent venous hemodialysis catheter inserted into the right jugular vein. In April 2013, the patient had been

Received on February 18, 2014 Revised on May 12, 2014

Accepted on May 19, 2014

Correspondence to: Won Suk Choi, Division of Infectious Diseases, Department of Internal Medicine, Korea University Ansan Hospital, Korea University College of Medicine, 123 Jeokgeum-ro, Danwon-gu, Ansan 425-707, Korea

Tel: +82-31-412-4271, Fax: +82-31-412-5984

E-mail: cmcws@hanmail.net

* No potential conflict of interest relevant to this article was reported. treated for infective endocarditis (IE) by Enterococcus faecium. The transesophageal echocardiography (TEE) performed at that time showed isoechoic oscillating filamentous mass attached to edematous prolapsed P3 of mitral valve and severe mitral regurgitation (grade IV). Surgery was recommended for the IE and severe mitral regurgitation, but the patient's family members declined due to the patient's age and poor general condition. The patient was treated with intravenous vancomycin for 6 weeks. During the present admission, the patient appeared ill with a temperature of $38.0^{\circ} \mathrm{C}$, a pulse rate of 94 beats per minute and blood pressure of 159/65 $\mathrm{mmHg}$. Laboratory data showed a C-reactive protein level of $15.9 \mathrm{mg} / \mathrm{dl}$ (normal $<3 \mathrm{mg} / \mathrm{dl}$ ) and leukocytosis of $10,480 / \mathrm{mm}^{3}$ and chest X-ray revealed mild pulmonary edema without any abnormal findings on the aortic arch (Fig. 1A). Peripheral blood culture and blood culture through the permanent venous hemodialysis catheter yielded methicillin resistant Stapylococcus aureus (MRSA). The hemocatheter was removed and a culture of the catheter tip also yielded MRSA. The automated system Vitek 2 revealed that the MRSA strain had a vancomycin minimum inhibitory concentration (MIC) of $\geq 0.5 \mathrm{ug} / \mathrm{ml}$, teicoplanin MIC of $\geq 0.5 \mathrm{ug} / \mathrm{ml}$, and linezolid MIC of $2 \mathrm{ug} / \mathrm{ml}$. Transthoracic echocardiography (TTE) showed moderate eccentric left ventricular hypertrophy, mitral valve prolapse and regurgitation (grade II), aortic valve regurgitation (grade II), and tricuspid valve regurgitation (grade II-III), all observed in prior 

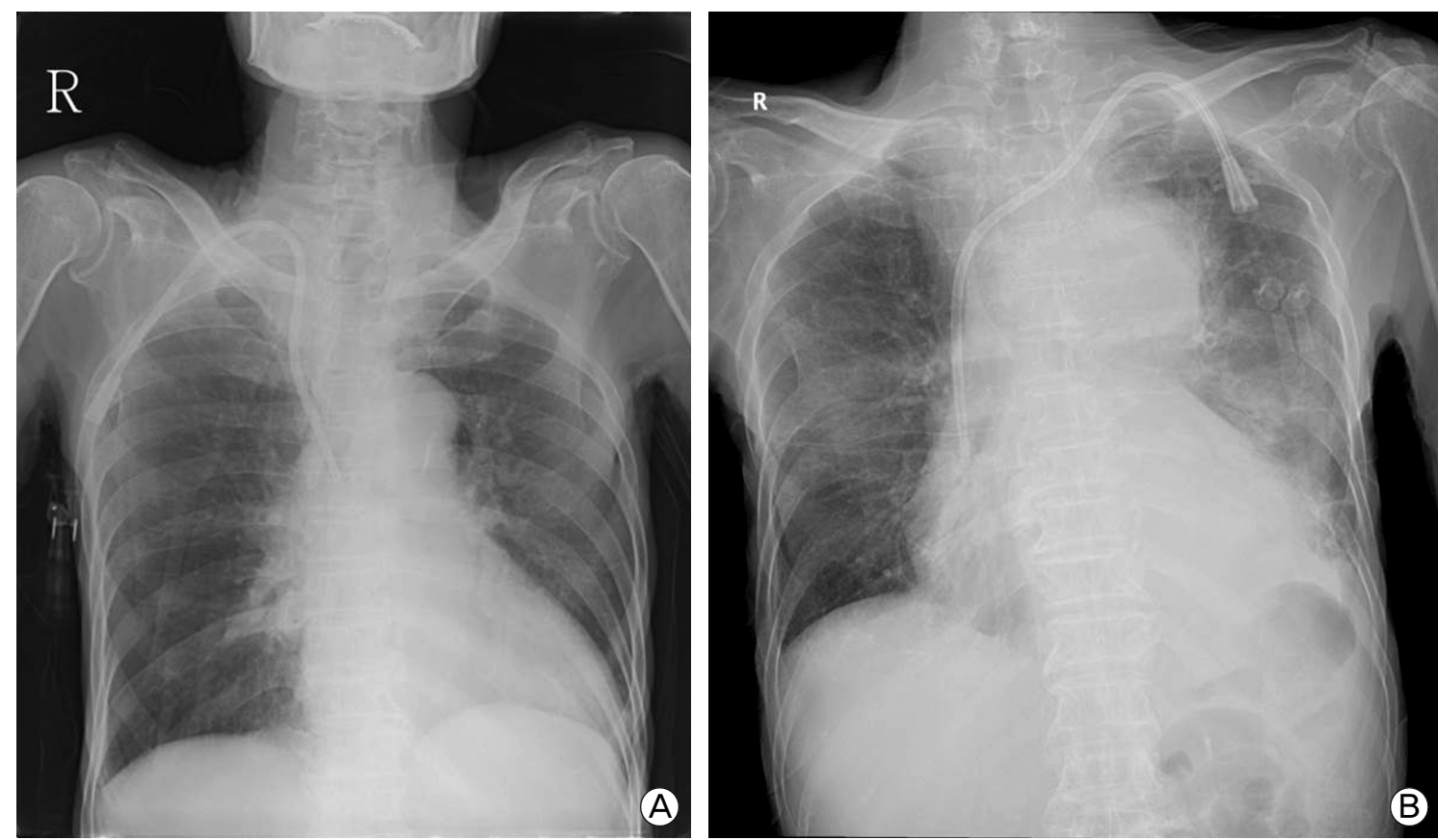

Fig. 1. Chest X-ray images. (A) A chest X-ray taken at admission shows mild pulmonary edema without any abnormal finding on the aortic arch. (B) A chest X-ray taken 2 weeks after admission shows bulging opacity on the aortic arch and predominant pulmonary edema on the left side.

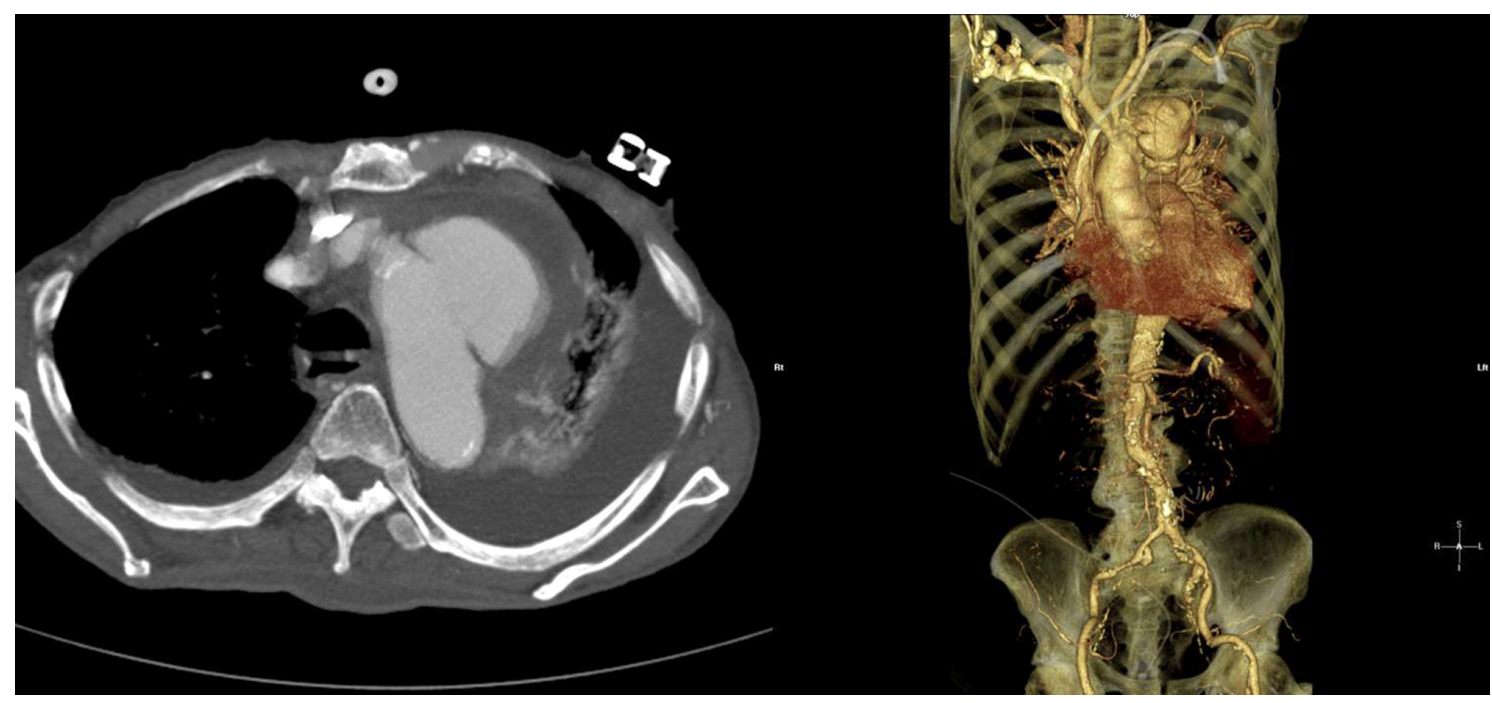

Fig. 2. Chest contrast-enhanced computed tomography scan images. Huge exophytix eccentric aortic aneurysm from the thoracic aorta, just distal to the left subclavian artery orifice is shown.

evaluations but with slight improvements compared to previous results. Hypermobile, hyperechogenic mass on P3 scallop of posterior mitral valve with moderate regurgitation was observed where the vegetation had existed previously. The patient began receiving intravenous vancomycin once admitted and the serum vancomycin trough concentration was maintained at 15 to 20 $\mathrm{ug} / \mathrm{ml}$. However, blood culture performed 10 days later still yielded MRSA. In response, antibiotics were changed from vancomy- cin to linezolid. Blood culture performed 4 days after initiation of linezolid showed no microorganism, but the size of the aortic arch had increased on a follow-up chest X-ray (Fig. 1B). Computed tomography scan of the chest showed huge exophytic eccentric aortic aneurysm from the thoracic aorta, just distal to the left subclavian artery orifice, seemingly in a ruptured state (Fig. 2). Surgery or endovascular treatment was recommended to remove the aortic aneurysm, but the patient's family members declined 
due to the patient's age and poor general condition as before. The patient was maintained with antibiotic treatment only and the size of the aneurysm increased rapidly, resulting in bronchial obstruction and superimposed pneumonia. On the 28th day of admission, the patient died of respiratory failure.

\section{Discussion}

Mycotic aneurysm is a life-threatening condition with significant morbidity and mortality. The reported hospital mortality rates range from $16 \%$ to $44 \%$.[3] The most common infection sites are the femoral artery and the abdominal aorta, followed by the thoraco-abdominal and thoracic aorta.[1,2] Mycotic aneurysm of the thoracic aorta is extremely rare, comprising approximately $0.65 \%$ to $1.3 \%$ of all aortic aneurysms.[4,5] As a result, only sporadic case reports of infected aneurysms of the aortic arch have been published. In the literature review, we could not find any case reports of mycotic aneurysm of the thoracic aorta in Korea.

The risk factors for infected aneurysm include arterial injury, antecedent infection, impaired immunity and atherosclerosis. [6, 7] Arterial injury is commonly due to intravenous drug use or iatrogenic mechanisms such as invasive monitoring. In the present case, the cause of the mycotic aortic aneurysm appeared to be arterial injury due to a permanent hemodialysis catheter insertion since MRSA was isolated from both the peripheral blood and the catheter tip. Although TTE was performed and there was no evidence of newly developed IE, IE could not be excluded completely because the TEE was not performed. Interestingly, the patient had a history of infective endocarditis by E. faecium three months ago, which could have facilitated the development of the mycotic aneurysm.

The diagnosis of mycotic aneurysm is based upon imaging the aneurysm and infection was confirmed by blood culture. CT angiography can be used to diagnose the aneurysm if specific features suggest infection.[8-10] In this case, CT scan was performed and the exophytic eccentric feature was compatible to an infected aneurysm.

The standard treatment for most mycotic aneurysms is prompt surgical intervention with antibiotic therapy, which is essential to control systemic sepsis and to achieve cardiovascular stability.[2] Antibiotic therapy alone is not sufficient to treat mycotic aneurysm because while antibiotic therapy may contain the infection, it cannot prevent complications such as rupture of the aneurysm.[1
1,12] In the present case, the patient's family members did not agree to surgery and the patient was maintained with antibiotic treatment only. The blood culture performed during antibiotic treatment showed no microorganism, but the aortic aneurysm increased in size and eventually obstructed the left bronchus.

In summary, we report a case with mycotic aneurysm located in the aortic arch, an extremely rare location for mycotic aneurysms. The patient was treated unsuccessfully with antibiotics alone.

\section{References}

1) Malouf JF, Chandrasekaran K, Orszulak TA: Mycotic aneurysms of the thoracic aorta: a diagnostic challenge. Am J Med 2003; 115: 489-96

2) Lee HL, Liu KH, Yang YJ, Kan CD: Bacteroides fragilis aortic arch pseudoaneurysm: case report with review. J Cardiothorac Surg 2008; 3: 29.

3) Hsu RB, Lin FY: Surgery for infected aneurysm of the aortic arch. The J Thorac Cardiovasc Surg 2007; 134: 1157-62.

4) Hsu RB, Lin FY: Infected aneurysm of the thoracic aorta. J Vasc Surg 2008; 47: 270-6.

5) Vallejo N, Picardo NE, Bourke P, Bicknell C, Cheshire NJ, et al: The changing management of primary mycotic aortic aneurysms. J Vasc Surg 2011; 54: 334-40.

6) Qureshi T, Hawrych AB, Hopkins NF: Mycotic aneurysm after percutaneous transluminal femoral artery angioplasty. J R Soc Med 1999; 92: 255-6.

7) Samore MH, Wessolossky MA, Lewis SM, Shubrooks SJ Jr, Karchmer AW: Frequency, risk factors, and outcome for bacteremia after percutaneous transluminal coronary angioplasty. Am J Cardiol 1997; 79: 873-7.

8) Azizi L, Henon A, Belkacem A, Monnier-Cholley L, Tubiana JM, Arrivé L: Infected aortic aneurysms: CT features. Abdom Imaging 2004; 29: 716-20.

9) Gomes MN, Choyke PL: Infected aortic aneurysms: CT diagnosis. J Cardiovasc Surg (Torino) 1992; 33: 684-9.

10) Vogelzang RL, Sohaey R: Infected aortic aneurysms: CT appearance. J Comput Assist Tomogr 1988; 12: 109-12.

11) Knosalla C, Weng Y, Yankah AC, Hofmeister J, Hetzer R: Using aortic allograft material to treat mycotic aneurysms of the thoracic aorta. Ann Thorac Surg 1996; 61: 1146-52.

12) Aliaga L, Cobo F, Miranda C, Lara J: Mycotic aneurysm of the aortic arch. Infection 2000; 28: 240-2. 